\title{
Giant left atrial myxoma hiding severe preoperative mitral regurgitation
}

\author{
Gregorio Laguna, Nuria Arce, Salvatore Di Stefano, Barbara Segura, \\ Miriam Blanco, Javier Castrodeza, Yolanda Carrascal \\ Department of Cardiac Surgery, Hospital Clínico Universitario, Valladolid, Spain
}

\section{Introduction}

Myxomas are the most common primary cardiac neoplasm. Usually, myxomas originate in the left atrium, and their implantation base is in the interatrial septum [1].

Large atrial myxomas can produce stenosis or mitral regurgitation. Generally, these mitral valve diseases tend to be reversible and disappear following the tumor resection [2], however, large myxomas with long-term evolution may permanently affect the mitral valve structure and may cause persisting damage of the valve after complete resection of the tumor [2-4].

At the same time, atrial myxomas may conceal or underestimate a preoperative mitral regurgitation, from any cause $[1,2,5,6]$.

\section{Case report}

A 68-year-old woman, without medical history or cardiovascular risk factors, was admitted with exercise-related syncope and dyspnoea which started the previous month. She did not present with nausea, vomiting, sweating, fever or any other symptoms. Physical examination showed a mitral diastolic murmur III/IV without presystolic murmur, without oedemas, skin lesions, or neurological focus.

Transthoracic echocardiogram (Fig. 1A) revealed a heterogeneous mass of $75 \times 52 \mathrm{~mm}$ in the left atrium, causing severe functional mitral stenosis without mitral regurgitation. The mass was prolapsing into the left ventricle during diastole. The left ventricular ejection fraction was preserved and there was evidence of severe pulmonary hyper- tension. The computed axial tomography showed a pedunculated mass on the left atrium, in close contact with the mitral valve annulus and mitral leaflets. It was anchored in the inferior part of the interatrial septum (Fig. 1B).

Cardiac surgery under cardiopulmonary bypass was performed. The mass, the implantation base and part of the interatrial septum, were resected through the right atriotomy (Fig. 1C). Subsequently, the septum was repaired with an autologous pericardium patch (Fig. 1D). The intraoperative transesophageal echocardiogram showed a severe central mitral regurgitation (Fig. 1E) with annular dilatation (Carpentier type I), thus it was repaired with a rigid mitral annuloplasty (number 30 Physio II ring) by the left atriotomy approach in the same procedure.

Postoperative period was uneventful. At discharge, the transthoracic echocardiogram revealed no mitral regurgitation or stenosis, and the definite anatomopathological exam confirmed the diagnosis of a cardiac myxoma.

\section{Discussion}

Left atrial myxomas, especially large ones with long-term evolution, may mechanically affect the mitral valve three-dimensional anatomy, by causing severe mitral stenosis or regurgitation due to a disturbance in the leaflet coaptation surface $[1,2,7]$. In most of cases, after a complete tumor resection, the mitral valve dysfunction disappears $[1,7]$.

However, the left atrial myxomas themselves, could cause a permanent mitral valve dysfunction: large myxomas, with a long-term evolution, by

Address for correspondence: Gregorio Laguna, MD, Department of Cardiac Surgery, Hospital Clínico Universitario, Ramón y Cajal Avenue, Postal Code: 47003, Valladolid, Spain, e-mail: goyotxmed@hotmail.com 


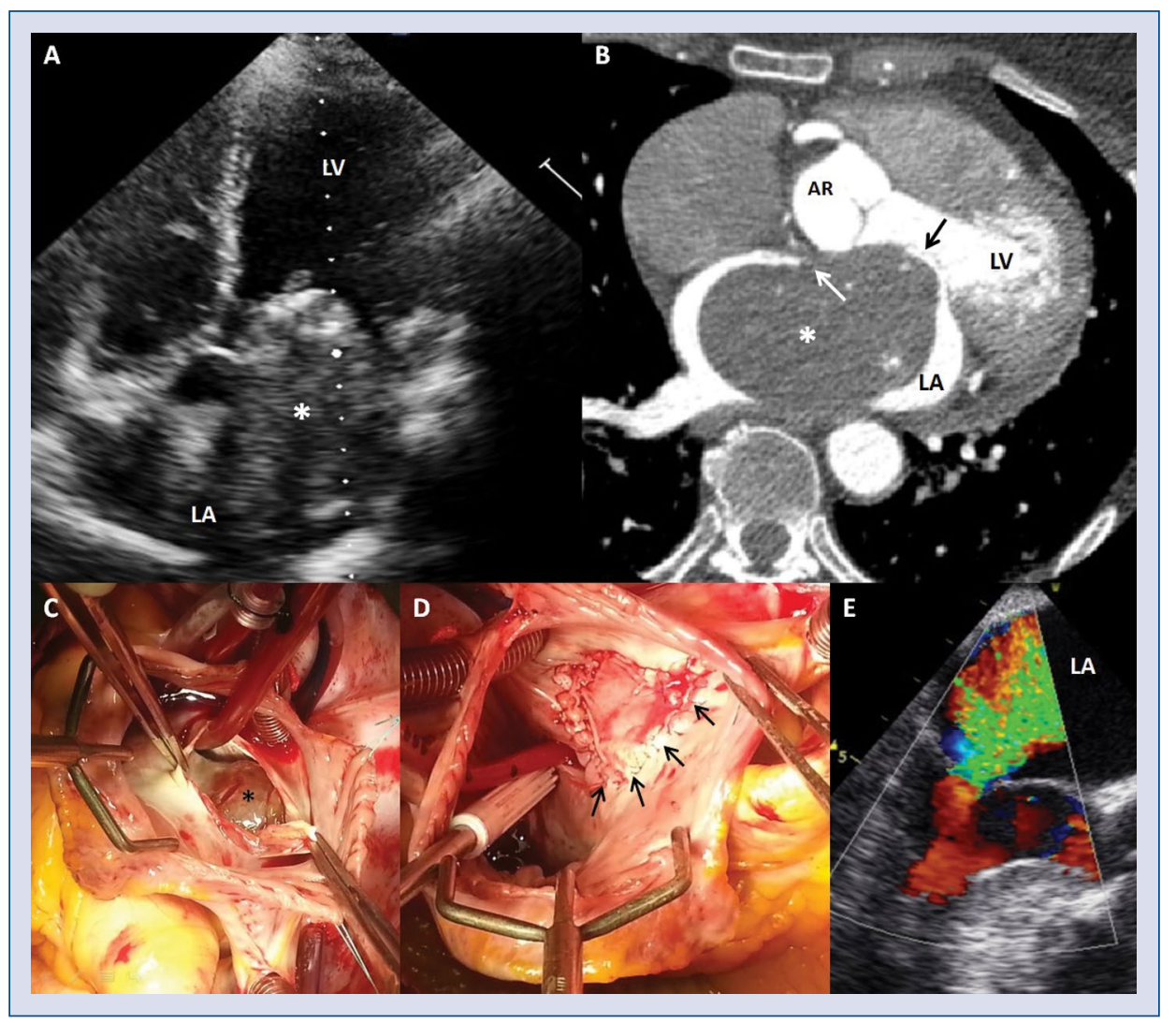

Figure 1. A. Transthoracic echocardiogram. Huge myxoma (asterisk); B. The computed axial tomography showing a large myxoma (asterisk) anchored to the interatrial septum (white arrow) in close contact with mitral valve leaflets (black arrow); C. Surgery image (asterisk) after opening the right atrium and interatrial septum; D. Sutured autologous pericardial patch to correct the defect after myxoma resection (black arrows); E. The intraoperative transesophageal echocardiogram reveals central severe mitral regurgitation; LA - left atrium; AR - aortic root; LV — left ventricle.

close contact with the valve leaflets, and a prolapse into the left ventricle can produce swelling of the mitral valve, distention or rupture of the chordae tendineae [2,3], and also mitral annulus dilatation $[5,8]$. In this way, valvular pathology is perpetuated (usually regurgitation caused by annular dilatation or prolapse) after complete myxoma resection [2-4].

Obviously, mitral valve dysfunction may be caused by other multiple etiologies like rheumatic disease, fibroelastic degeneration, Barlow disease, among others. In these cases, despite a complete tumor resection, it is hard to confirm the disappearance of valve dysfunction [9].

Moreover, large left atrial myxomas, may conceal or underestimate a significant preoperative regurgitation from any etiology $[1,4-6,10]$, because they take up significant space in the left atrium. Myxomas might camouflage an easily hidden mitral regurgitation caused by annular dilatation other than the one generated by a prolapse of mitral leaflets [4]. Some scientific reports show that myxomas with short pedicles and wide implantation bases in lower interatrial septum areas, would easily hide a mitral regurgitation [10], as they are less mobile and they better resist the direct pressure of regurgitation volume.

Preoperative echocardiogram is essential in assessing the severity and mechanism of mitral regurgitation, however, in the presence of a left atrial mass, the diagnostic sensitivity is much lower [5].

Several authors have reported the association between mitral regurgitation and myxomas [11], emphasizing the importance of an intraoperative transesophageal echocardiogram study to discover the mechanism and severity of mitral regurgitation, that can remain after resection of the mass $[4,6,10,11]$. Persisting mitral regurgitation after resection of cardiac myxoma must be approached concurrently in order to achieve an optimal result, either by repairing or replacing the mitral valve to ensure a good long-term patient prognosis.

Conflict of interest: None declared 


\section{References}

1. Germing A, Lindstaedt M, Mügge A, et al. Severity of mitral regurgitation may be underestimated in the presence of a left atrial myxoma. J Heart Valve Dis. 2006; 15(6): 830-832, indexed in Pubmed: 17152792.

2. Kumar B, Raj R, Jayant A, et al. Left atrial myxoma, ruptured chordae tendinae causing mitral regurgitation and coronary artery disease. Ann Card Anaesth. 2014; 17(2): 133-136, doi: 10.4103/0971-9784.129850, indexed in Pubmed: 24732613.

3. Snir E, Caspi A, Vidne BA. Rupture of chordae tendineae associated myxoma of the left atrium. Scand J Thorac Cardiovasc Surg. 1985; 19(2): 189-191, indexed in Pubmed: 4048892.

4. Yamaguchi K, Koide Y. Role of intraoperative transesophageal echocardiography in detecting masked mitral regurgitation during left atrial myxoma surgery. J Anesth. 2015; 29(1): 134-137, doi: 10.1007/s00540-014-1885-8, indexed in Pubmed: 25056257.

5. Vinasco DMO, Sánchez MA, Esquivel JEO. Insuficiencia mitral severa posresección de mixoma auricular gigante: presentación de un caso y revisión de la literatura. Revista Española de Anestesiología y Reanimación. 2013; 60(7): 403-406, doi: 10.1016/j.redar.2012.05.024.
6. Formica F, Sangalli F, Paolini G. Unusually large left atrial myxoma causing mitral valve occlusion and hiding a severe mitral regurgitation: a case report. Heart Surg Forum. 2006; 9(6): E849-E850, doi: 10.1532/HSF98.20061077, indexed in Pubmed: 17060039.

7. Gadhinglajkar S, Sreedhar R. Utility of transoesophageal echocardiography during surgery on left atrial myxoma. Ann Card Anaesth. 2008; 11(2): 142-143, indexed in Pubmed: 18603766.

8. López Almodóvar LF, Lima P, Buendía JA, et al. Giant left atrial myxoma and type I severe mitral valve regurgitation. Eur J Cardiothorac Surg. 2008; 33(6): 1143, doi: 10.1016/j. ejcts.2008.02.028, indexed in Pubmed: 18374591.

9. Mazuz M, Pandian N, Kerber R. Left atrial myxoma and unrelated mitral valve disease. J Am Coll Cardiol. 1983; 1(4): 1170-1173, indexed in Pubmed: 6833656.

10. Teng YH, Wang CW, Mao CC. Underdiagnosis of the severity of mitral regurgitation in left atrial myxoma. J Heart Valve Dis. 2010; 19(6): 806-807, indexed in Pubmed: 21214112.

11. Selkane C, Amahzoune B, Chavanis N, et al. Changing management of cardiac myxoma based on a series of 40 cases with long-term follow-up. Ann Thorac Surg. 2003; 76(6): 1935-1938, indexed in Pubmed: 14667616. 